\title{
Evaluación del impacto de la vinculación con la sociedad en el caso de la carrera de Odontología de la Universidad Católica de Cuenca
}

\author{
Evaluation of the impact of linkage with society in the case of the Odontology \\ career of the Catholic University of Cuenca
}

\begin{abstract}
Jaime Ramiro Quezada Ortega. ${ }^{1}$, Christian Mauricio Banegas Campoverde. ${ }^{2} \&$ Yonimiler Castillo Ortega. ${ }^{3}$
\end{abstract}

\begin{abstract}
.
DOI: https://doi.org/10.33262/concienciadigital.v4i1.1.1550
\end{abstract}

Introduction. The projects of connection with society, implemented by Ecuadorian universities, are an opportunity of service, and an alternative of approaching their students with society, which will allow them to feel the reality of social problems and be able to propose or provide solutions to them, beyond being the link a mere fulfillment of obligations raised by the institutions of control of higher education. But for these projects to fulfill the service function and respond to the needs of society, they must be evaluated, to make corrections or adjustments if necessary, to guarantee the success of the projects. Objective. Apply a set of indicators that measure the impact of the process of Relationship with the Society of the Dentistry career of the Catholic University of Cuenca. Methodology. The research design is analytical, descriptive, and quali-quantitative of a non-experimental type. The sample is non-probabilistic for convenience, two data collection sheets were made, for the managers and for the beneficiaries with three indicators each and two structured interviews. Results. The project improved the quality of life of $66 \%$ of the patients, the care provided was very good (58.33\%), this type of project is very useful for the community $(91.67 \%)$ in terms of planning as well very good (52.94\%), fulfillment of the objectives $(64.71 \%)$, in the level of citizen participation indicator, the project was planned jointly between the university and the beneficiary institution, following up until its completion. Conclusion. It was concluded on the importance of carrying out

\footnotetext{
${ }^{1}$ Universidad Católica de Cuenca, Docente de la Carrera de Arquitectura, Posgrado, Maestría en Desarrollo Local Mención en Ordenamiento Territorial, Cuenca, Ecuador, jquezada@ucacue.edu.ec, https://orcid.org/0000-0002-5599-7229

${ }^{2}$ Universidad Católica de Cuenca, Docente de la Carrera de Economía, Cuenca, Ecuador, cbanegas@ucacue.edu.ec, https://orcid.org/0000-0003-1102-2283

3 Universidad Católica de Cuenca, Posgrado, Director de la Maestría en Desarrollo Local Mención en Ordenamiento Territorial. Director de la Carrera de Economía, Cuenca, Ecuador, ycastilloo@ucacue.edu.ec https://orcid.org/0000-0002-7710-5199
} 
evaluation processes of the projects of connection with the society and the evaluated project had a positive impact on the beneficiaries.

Keywords: Relationship with society - university - society

\section{Resumen}

Introducción. Los proyectos de vinculación con la sociedad, implementados por las universidades ecuatorianas, son una oportunidad de servicio, y una alternativa de acercamiento de sus estudiantes con la sociedad, lo que les permitirá, palpar la realidad de las problemáticas sociales y poder plantear o brindar soluciones a las mismas, más allá de ser la vinculación un mero cumplimiento de obligaciones planteadas por las instituciones de control de la educación superior. Pero para que estos proyectos cumplan con la función de servicio y dar respuesta a las necesidades de la sociedad, deben ser evaluados, para tomar los correctivos o ajustes de ser necesario, para garantizar el éxito de los proyectos. Objetivo. Aplicar un conjunto de indicadores que midan el impacto del proceso de Vinculación con la Sociedad de la carrera de Odontología de la Universidad Católica de Cuenca. Metodología. El diseño de investigación es analítica, descriptiva, y cuali-cuantitativa de tipo no experimental. La muestra es no probabilística por conveniencia, se realizaron dos fichas de recolección de datos, para los gestores y para los beneficiarios con tres indicadores cada una y dos entrevistas estructuradas. Resultados. El proyecto mejoró la calidad de vida del 66\% de los pacientes, la atención brindada fue muy buena $(58,33 \%)$, este tipo de proyectos son de gran utilidad para la comunidad $(91,67 \%)$ en cuanto a su planificación también muy buena $(52,94 \%)$, cumplimiento de los objetivos $(64.71 \%)$, en el indicador de nivel de participación ciudadana, se planifico el proyecto conjuntamente entre la universidad y la institución beneficiaria, dando un seguimiento hasta la finalización del mismo. Conclusión. Se concluyó en la importancia de realizar procesos de evaluación de los proyectos de vinculación con la sociedad y el proyecto evaluado tuvo un impacto positivo en los beneficiarios.

Palabras clave: Vinculación con la sociedad - universidad - sociedad

\section{Introducción}

En la actual dinámica del mundo globalizado, la universidad, tal como lo manifiesta Pérez (2017) toma un protagonismo fundamental en el desarrollo de las sociedades, ya que es el ente encargado de la formación de profesionales que deberán repercutir e incidir en los procesos sociales, que potencialicen el bienestar de las sociedades a las que se pertenecen. Es ahí donde tienen vital importancia los programas y proyectos de vinculación con la sociedad, cuyos conceptos y concepciones a nivel de América Latina, tienen diferentes denominaciones, tal como lo manifiestan Castro, Borroto, y Almuiñas (2018) ya que estas dependen de posturas ideológicas y políticas, y de las relaciones entre la universidad y la sociedad, organizaciones e instituciones, así se denominan: vinculación con la colectividad, extensión universitaria y difusión cultural, difusión cultural y extensión de los servicios, extensión universitaria, vinculación con la sociedad.

El ir mejorando las herramientas de vinculación con la sociedad, es una tarea permanente de todas las universidades, ya que es ese, uno de los espacios en los cuales sus estudiantes 
próximos a graduarse pondrán a prueba sus conocimientos, generando y brindando posibles soluciones que mitiguen la problemática de las comunidades, a las cuales esperan servir en un futuro cercano como profesionales, teniendo siempre el horizonte de la relación UniversidadSociedad.

En este sentido en el Ecuador en los años 80 y 90, Simbaña y Correa (2017) manifiestan que se produce un cambio en la extensión universitaria, hoy conocida como vinculación con la sociedad (VcS), ya que fue incorporada a la normativa legal y estatutaria de las universidades, evidenciando además un desfase entre la universidad ecuatoriana y la sociedad, ya que mientras el país requería con urgencia de avances científicos y tecnológicos, la universidad continuaba impartiendo una formación enciclopédica y generalista, con la excepción de pocas carreras que intentaban de una u otra forma vincularse con la sociedad, en sus proyectos de extensión universitaria. Además, Maldonado, Buenaño, \& Benavides (2018) manifiestan que la evaluación de las instituciones de educación superior (IES) llevada a cabo por las instancias gubernamentales en el 2008, permitió develar la decadencia de algunas prácticas dentro de las IES, con respecto a la calidad de la educación, lo que conllevó a una participación directa del gobierno ecuatoriano en el sistema de educación superior.

En el Ecuador desde el año 2010, con la implementación de la Ley Orgánica de Educación Superior (LOES) donde se señala como obligatoriedad a las universidades su vinculación de diferentes formas con sus comunidades, y ratificada en el Plan Nacional del Buen Vivir (20172021) en el "Eje 1: Derechos para todos durante toda una vida", que manifiesta que el sistema de educación superior del país debe posicionarse como un referente en la región, sobre todo enfocándose en la consecución de los Objetivos Nacionales de Desarrollo, donde deberá existir una verdadera vinculación de los estudiantes universitarios, ya que las universidades tal como lo manifiesta Mendoza, Jácome y Vélez (2017) las universidades están llamadas a responder por el desarrollo de los sectores sociales donde realizan sus actividades, mediante la prestación de servicios a la comunidad a la que se pertenecen. En este sentido Viña, Sacoto y Landívar (2019) manifiestan que estos servicios deberán estar dentro de un marco legal, donde prevalezcan la calidad, innovación y pertinencia. Así, Malagón (2006) alega que las universidades han tenido que modernizar y flexibilizar sus estructuras organizativas, adecuando sus misiones a los intereses y demandas de la sociedad.

Además de la $\mathrm{VcS}$, la universidad tiene como ejes fundamentales, la investigación y la docencia. Y de estas tres funciones la $\mathrm{VcS}$ es el eje principal en la cual se desenvuelven las otras dos, tal como lo plantea De Aparicio, Chininin, y Toledo (2017). De la misma manera Barreno, Barreno y Olmedo (2018) manifiestan que éste será el vínculo desde la academia, con la parte práctica-teórica que le permita al estudiante su inclusión en el mercado laboral y también le permita a la universidad actualizar los perfiles profesionales de sus carreras.

También, Serrano y Condor (2018) y Álava, Moreira y Viteri (2019) coinciden en afirmar que dicho contacto le permitirá al estudiante reflexionar, sobre las profundas desigualdades que limitan que todos los pobladores de una región, tengan igual oportunidad de acceso a los bienes y servicios, y a decir de Cedeño, Ostaíza, Vélez y Solórzano (2018) los universitarios deben adquirir una serie de atributos que los conviertan en seres competentes al servicio de la 
humanidad, y que esta vinculación además tal como lo manifiesta Hermida (2017) tiene que ser una vinculación intercultural con la sociedad y sumak kawsay donde la cultura es entendida como las costumbres, conocimientos, creencias, interpretaciones, creaciones, producciones y construcciones de los pueblos o nacionalidades del Ecuador, en búsqueda de potenciar su desarrollo. Según los criterios de Herrera (2016), la participación universitaria se ha vuelto clave en las estrategias de desarrollo, todo ello dentro de los cambios que a nivel mundial han surgido en el ámbito de la ciencia y la técnica, enmarcadas dentro de la academia.

En este contexto el caso ecuatoriano no es una excepción, en un mundo globalizado donde la competitividad depende cada día más del uso del conocimiento y las innovaciones tecnológicas, Quezada Carmenate y Brito (2017) manifiestan que la riqueza de un país está en el cultivo de la inteligencia de su pueblo, y es ahí donde las universidades ecuatorianas juegan un papel preponderante en el desarrollo de la comunidad.

Por ello, las universidades ecuatorianas, tal como lo manifiestan Brito, Gordillo y Quezada (2016) requieren de modelos que permitan garantizar la investigación, su oferta académica y plantear soluciones a las necesidades de la comunidad local, regional y nacional, en este mismo sentido Rueda, Acosta y Cueva (2020) plantean; que cuando estos proyectos universitarios llevan a la sociedad el conocimiento que desarrollan las universidades, lo que están realizando es una transferencia de conocimientos y tecnología, que permitan a las universidades plantear soluciones o satisfacer las necesidades de la comunidad, para lo cual se deberán generar programas y proyectos que sean de interés público.

Brito, Quezada y Gordillo (2018a) además manifiesta, que estos proyectos deben tener un carácter de sostenibilidad y preferiblemente ser realizados por un equipo multidisciplinar, que respondan a las complejas y dinámicas territoriales, ya que la universidad debe llevar los conocimientos a las comunidades, para potenciar sus teorías y conceptos científicos, técnicos y humanísticos, que le permitan generar nuevos saberes, tomando en consideración los saberes ancestrales presentes en las comunidades de su intervención.

En el sentido de la sostenibilidad Pérez (2018) acota además que, para entender una educación para el desarrollo sostenible, es necesario que se implementen principios y estrategias de enseñanza participativas en sus propuestas, y que tengan objetivos como los de la equidad intergeneracional, equidad de género, la tolerancia, la paz, reducción de la pobreza, cuidado del medio ambiente, conservación de los recursos naturales y la justicia social, conocimientos que permitirán satisfacer necesidades de desarrollo local. En este mismo sentido las Naciones Unidas en su Agenda 2030 para el desarrollo sostenible, aprobada en el 2015, hace un llamamiento para que la comunidad académica, contribuya estrechamente en el desarrollo local de sus comunidades (ONU,2015).

Además, Brito, Quezada y Gordillo (2018a) afirma que estos proyectos deben partir desde un plan de desarrollo institucional, de la pertinencia de las carreras que pertenecen a la universidad conjuntamente con los planes de desarrollo y ordenamiento territorial (PDOT) de los diferentes niveles de gobierno, enmarcados en el plan nacional de desarrollo, que en resumidas cuentas es desde donde deben nacer dichos programas y proyectos de $\mathrm{VcS}$, por parte de las universidades. 
Y para ello Riofrío, Serrano y Sotomayor (2018) manifiestan que las instituciones de educación superior deben mejorar sus estructuras y organizaciones, con las que han venido trabajando, para que las respuestas a las necesidades de las comunidades sean más eficientes, en donde las tecnologías de la información y comunicación (TIC) juegan un rol importante, en una sociedad donde sus entornos están altamente tecnificados, y éstas nuevas tecnologías, indudablemente generan nuevas competencias en estudiantes y profesores.

Éstas nuevas posibilidades de formación y de trabajo de la cual hablan Riofrío, Serrano \& Sotomayor se vuelven hoy más necesarias que nunca, con la aparición de la pandemia del covid 19, es notoria la importancia de las redes sociales o del teletrabajo y del uso de los medios tecnológicos en la formación académica universitaria, caracterizada sobre todo por la velocidad con la que se transmite la información.

A pesar de todo lo anteriormente manifestado no se han logrado todavía realizar una evaluación de los proyectos de investigación desde la óptica de sus involucrados, es decir los estudiantes y sus tutores y las comunidades y sociedades a las que están articulados dichos proyectos.

En cuanto al modelo de gestión a implementar para la evaluación de los proyectos de vinculación con la sociedad, Chávez (2016) manifiesta que no existe un modelo de gestión perfecto, sin embargo, plantea algunas características que debería tener dicho modelo, como son:

- Participación de la universidad de manera crítica, planificada y dinámica, de manera conjunta con organizaciones comunitarias, empresariales, culturales y sociales, además de veedores externos, que permitan una participación y adaptación al entorno productivo y social.

- Un ente gubernamental de la universidad, que tenga representación y participación de las diferentes unidades académicas que lo conforman y con capacidad de reprogramar y revisar sus planes y proyectos de $\mathrm{VcS}$.

- Adecuado financiamiento que además podría contar con recursos externos, que permitan la viabilidad de los proyectos de $\mathrm{VcS}$.

A estas características habría que señalar, además, que para que cualquier modelo de gestión que se proponga pueda tener el éxito deseado, es necesario, como lo describen Castro, Borroto, y Almuiñas (2018) que las universidades de una misma localidad deberían unirse, antes que competir, para articularse y fortalecer su participación en el sistema educativo nacional e internacional, como productoras de ciencia y conocimientos que partan de la experiencia y reflexiones de sus investigaciones, además de adecuar sus agendas de pregrado y posgrado, de investigación y de $\mathrm{VcS}$, para fortalecer el desarrollo social de la economía de las localidades a las que se deben. En este mismo sentido Brito, Quezada y Gordillo (2018 b) plantea que la VcS debe tener un papel articulador entre las diferentes sujetos e instancias de la universidad, entre las otras IES y los grupos y organismos sociales para compartir e intercambiar ideas, conocimientos y proyectos.

\section{Metodología}


Utilizando una metodología analítica, descriptiva, cualitativa con la aplicación de entrevistas estructuradas para los funcionarios y directivos de la institución beneficiaria y del proyecto de la universidad y cuantitativa con la aplicación de cuestionarios para los beneficiarios y estudiantes participantes del proyecto de vinculación con la sociedad, de carácter no experimental, ya que se la ejecutó luego de la culminación del proyecto de vinculación en la institución beneficiaria, además para la recolección de información de los pacientes, se realizó una muestra no probabilística por conveniencia, con lo cual obtuvimos información primaria, y mediante el método analítico sintético, valorar de manera global, el nivel de impacto del proyecto de vinculación con la sociedad

Para identificar el marco jurídico de la metodología de vinculación con la sociedad de la universidad ecuatoriana, se realizó una revisión documental, del marco jurídico ecuatoriano y de las normativas y procesos que articula la Universidad Católica de Cuenca, para la ejecución de sus proyectos de vinculación con la sociedad, los cuales se encuentran en su página web perfectamente definidos en unos formatos, a realizar durante la planificación y ejecución del proceso de vinculación de los proyectos.

En cuanto a los indicadores que nos permitan medir el impacto de los procesos de vinculación con la sociedad de la carrera de Odontología de la Universidad Católica de Cuenca, se definieron de los indicadores planteados por Del Cioppo y Bello, aquellos relacionados con comportamientos asociados a valores organizacionales, estos se justifican ya que se los analizan a nivel de la organización, de las estrategias y metas del proyecto propuesto.

Finalmente, estos indicadores fueron validados, en la evaluación del proyecto de vinculación de la carrera de odontología, denominado "Mejoramiento de la salud bucal del paciente con discapacidad, implementado en ADINEA, en la ciudad de Cuenca".

\section{Resultados}

En cuanto a la evaluación de impactos que aplica la Universidad Católica de Cuenca, ésta emplea un documento denominado "“Planificación de Evaluación de Impacto", en la cual se realiza un análisis de la situación antes de iniciar con el proyecto de vinculación, la identificación de los beneficiarios del proyecto, tanto directos como indirectos, y los objetivos específicos que deberán estar articulados al general, además de identificar de manera precisa, cuáles serán las actividades que se realizaran para alcanzar cada uno de los objetivos, esta evaluación se realiza conjuntamente con la ejecución del proyecto, faltando realizar una evaluación posterior a la fecha de terminación de los proyectos, lo que nos permitirá tener una valoración real del impacto generado por los mismos en las comunidades o instituciones implementadas. Tomando en cuenta la diversidad de proyectos, de las diferentes carreras que posee la universidad, estos serán valorados de manera individual, en procura de medir, por un lado, el nivel de colaboración tanto de organizaciones y comunidades con la universidad, y por otro, las actividades que ésta utiliza, para dar respuesta a las necesidades de las comunidades de su entorno y de la sociedad.

La carrera de Odontología de la Universidad Católica de Cuenca, desde varios años atrás a mantenido programas y proyectos de vinculación con la sociedad, en procura de brindar 
servicios odontológicos a grupos de atención prioritaria sobre todo de las parroquias rurales de la provincia del Azuay y Cañar, como los que actualmente están en marcha: "Juntos por una sonrisa: Este proyecto tiene como impacto contribuir a la disminución de los riesgos de enfermedades relacionadas al estado nutricional de los niños de 0 a 12 años de edad en la Provincia del Cañar", "Creando sonrisas en niños, niñas y adultos de las parroquias de la provincia del Azuay. 2019 - 2021 Prefectura del Azuay" o "Atención bucodental en comunidades beneficiarias de las parroquias urbanas del programa "Sonríe Cuenca" 2019 2021 Ministerio de Salud Pública - Coordinación Zonal 6.", en este sentido el proyecto estudiado en la presente investigación: Mejoramiento de la salud bucal del paciente con discapacidad, se enmarca en la atención a grupos prioritarios de nuestra sociedad, que además han permitido a los estudiantes tener un contacto directo con pacientes de escasos recursos económicos y poder aportar al bienestar social de su región.

Para los indicadores en la evaluación de un proyecto, estos deben medir las estrategias, tal como lo manifiesta Torres, Escalante, y García (2020), "Los indicadores sirven para medir la efectividad de las estrategias de vinculación con el entorno y en consecuencia desarrollar nuevas o mejorar las existentes". La universidad no alcanzaría el éxito deseado si no cuenta con un plan de evaluación a ser implementado en la vinculación de la universidad-empresacomunidad, de ahí que esta investigación utilizó un método mixto concurrente según Creswell (2019), donde se brinda prioridad a la recolección y análisis de los datos tanto cualitativos como cuantitativos, dentro de un mismo estudio que plantea un conjunto de indicadores de vinculación de fácil comprensión, que nos permitan valorar el proyecto de vinculación, esto nos permitirá a corto plazo mejorar las propuestas de proyectos de vinculación, en procura de que éstos tengan los alcances deseados, y sobre todo brinden soluciones desde la academia a las diferentes problemáticas por la que atraviesa nuestra sociedad.

Algunas universidades del Ecuador, han realizado evaluaciones a sus programas y proyectos de VcS implementados, para analizar si los mismos están cumpliendo con sus objetivos, entre ellas tenemos los realizadas por Mendoza, Jácome y Vélez (2017) a la Pontificia Universidad Católica del Ecuador sede Esmeraldas (PUCE-Esmeraldas) correspondiente al año académico 2015-2016, en el período comprendido entre febrero y agosto de 2016, en la cual participaron directivos, jefes departamentales, docentes y estudiantes, para dicha investigación se aplicó un estudio cuali-cuantitativo, analítico sintético, cuyos resultados obtenidos nos demuestran que los programas y proyectos de vinculación, tienen debilidades tanto en su estructuración, como en la articulación con la docencia y la investigación, lo que ha generado la falta de interés y poca participación por parte de estudiantes y docentes. El estudio también revela, que, a criterio de los docentes, existen proyectos interesantes, sin embargo, estos son aislados y repetitivos. Finalmente cabe recalcar que los estudiantes, tienen el concepto de que los proyectos de vinculación son ayudas de carácter social y no de beneficio mutuo.

Además, para poder analizar el nivel de impacto de los proyectos de vinculación implementados por las IES, es importante tomar en consideración algunas variables propuestas por el Observatorio Iberoamericano de la Ciencia, la Tecnología y la Sociedad (OCTS-OEI), y la Red Iberoamericana de Indicadores de Ciencia y Tecnología (RICYT) en el Manual Iberoamericano de Indicadores de Vinculación de la Universidad con el Entorno Socioeconómico, más 
conocido como Manual de Valencia (2017), el mismo que es el producto de un largo proceso de reflexiones realizadas desde el año 1995, con la colaboración de expertos, universidades y organismos internacionales. En lo referente a los indicadores de medición (p.14), distingue tres tipos:

- indicadores de actividad

- indicadores de resultados

- indicadores de impacto

En este mismo sentido Del Cioppo y Bello (2018) en "Indicadores de impacto social para evaluación de proyectos de vinculación con la colectividad" proponen el diseño de un modelo de evaluación basado en algunos criterios de la Asociación Internacional para la Evaluación de Impactos (IAIA), que plantea que la evaluación de los impactos, nos permiten diseñar y proponer mejores políticas, programas y proyectos, para enfrentar los grandes desafíos y conflictos por recursos cada vez más escasos, conjuntamente con desigualdades en diferentes ámbitos. Este modelo, fue realizado para evaluar el impacto de los indicadores de los proyectos de vinculación con la colectividad, de Facultad de Economía Agrícola de la Universidad Agraria del Ecuador en el año 2017, sin embargo, el autor propone que puede ser utilizado por otras IES, modelo que además se enfoca en los lineamientos estructurados para la evaluación y gestión de impactos sociales de proyectos, vigente desde abril de 2015 y desarrollado por la Asociación Internacional para la Evaluación de Impactos.

La propuesta de los indicadores para la evaluación del impacto de los proyectos de $\mathrm{VcS}$, de los autores se divide en tres grandes variables:

1. Impacto en los Beneficiarios de los proyectos de labor comunitaria; dentro del cual tenemos:

- La participación ciudadana, que a su vez tiene siete indicadores

1.-Percepción y aprobación de la gestión

2.-Percepción de la institucionalidad

3.-Satisfacción con los resultados y la puesta en práctica

4.-Integración comunitaria a través de la asistencia y participación

5.-Ausentismo y deserción

6.-Apropiación y disposición social para la participación

7.-Participación en el seguimiento y mecanismos de vigilancia

- Desarrollo formativo, que comprende tres indicadores

1.-Mejoramiento de la capacidad de la formación

2.-Incremento de los indicadores de situación laboral

3.-Competencias profesionales y técnicas

- Educación continua, que analiza los siguientes cuatro indicadores

1.-Capacidades y potencialidades de la ciudadanía

2.-Acreditación de programas

3.-Certificación de procesos

4.-Percepción del proyecto de formación y diseño de aprendizaje 
2. Impacto en las familias y comunidad, dentro del que tenemos:

- Bienestar social, que analiza cuatro indicadores:

1.-Calidad de entorno

2.-Mejoramiento y diversificación de las actividades sociales

3.-Equidad de género e integración

4.-Naturaleza de conciencia social

- Creación de valores económicos, que analiza tres indicadores:

1.-Mejora en las capacidades del grupo familiar

2.-Distribución y diversificación de ingresos

3.-Estandares de vida material y relaciones sociales

3. Impacto en los gestores del proyecto, dentro del cual tenemos:

- Rendimiento de los grupos estudiantiles, donde analiza dos indicadores:

1.-Integración estudiantil con los proyectos comunitarios

2.-Percepción de los estudiantes con respecto a la aplicación y puesta en marcha

- Percepción del profesorado, que igualmente analiza dos indicadores:

1.-Desarrollo académico de estrategias, técnicas y gestión

2.-Aspectos psicológicos, evolutivos y trabajo en equipo

Por lo que la presente investigación propone aplicar algunos indicadores, que permitan una valoración del proyecto luego de su ejecución, con lo cual se podrá medir, sobre todo a nivel de los beneficiarios, cuál es el impacto que el proyecto generó en su bienestar personal y comunitario, esto nos permitirá tomar los correctivos de así requerirlo en el proyecto a futuro.

A partir del modelo de evaluación propuesto por Del Cioppo y Bello, se identificaron los indicadores en una matriz de determinación de necesidades de información, para ser implementada en el caso de la Carrera de Odontología de la Universidad Católica de Cuenca, en el proyecto de $\mathrm{VcS}$ denominado: Mejoramiento de la salud bucal del paciente con discapacidad, que es la siguiente:

\section{Tabla 1}

Matriz de determinación de necesidades de información

\begin{tabular}{|c|c|c|c|}
\hline $\begin{array}{l}\text { Problema de la } \\
\text { Investigación }\end{array}$ & $\begin{array}{l}\text { Actores } \\
\text { del } \\
\text { Proyecto }\end{array}$ & Necesidades de Información - Indicadores & $\begin{array}{l}\text { Instrumento de } \\
\text { recolección de } \\
\text { la Información }\end{array}$ \\
\hline \multirow{3}{*}{$\begin{array}{l}\text { ¿Cómo medir } \\
\text { el impacto del } \\
\text { proceso de } \\
\text { Vinculación } \\
\text { con la } \\
\text { Sociedad en el } \\
\text { caso de la } \\
\text { carrera de } \\
\text { Odontología de } \\
\text { la Universidad } \\
\text { Católica de } \\
\text { Cuenca? }\end{array}$} & \multirow{2}{*}{$\begin{array}{l}\text { Beneficiari } \\
\text { os directos } \\
\text { del } \\
\text { proyecto: } \\
\text { Institución }\end{array}$} & \multirow{2}{*}{$\begin{array}{l}\text {-Nivel de participación ciudadana (Asistencia, } \\
\text { socialización, participación en el seguimiento, } \\
\text { acceso a la información) } \\
\text {-Organización (Talleres, atención de los } \\
\text { gestores, organización de actividades) } \\
\text {-Utilidad y valor agregado (Bienestar familiar) } \\
\text {-Expectativas (influencia positiva) }\end{array}$} & $\begin{array}{l}\text { Directivos: } \\
\text { Entrevista }\end{array}$ \\
\hline & & & $\begin{array}{l}\text { Pacientes: } \\
\text { Cuestionario }\end{array}$ \\
\hline & $\begin{array}{l}\text { Gestores } \\
\text { del } \\
\text { proyecto: } \\
\text { estudiante }\end{array}$ & $\begin{array}{l}\text {-Integración con la comunidad (Talleres, } \\
\text { recepción de opiniones) } \\
\text {-Percepción respecto a la planificación y } \\
\text { ejecución del proyecto (Satisfacción) } \\
\text {-Metas y resultados }\end{array}$ & $\begin{array}{l}\text { Estudiantes: } \\
\text { Cuestionario }\end{array}$ \\
\hline
\end{tabular}


ISSN: 2600-5859

\begin{tabular}{|c|l|l}
\hline s y & -Estrategias, técnica y gestión académica & Directora: \\
directora & -Trabajo en equipo & Entrevista \\
\hline
\end{tabular}

Fuente: Elaboración propia.

Con la definición de los diferentes actores y los indicadores planteados en la tabla 1, se diseñaron los instrumentos de recolección de información, definiendo para los beneficiarios (pacientes) y estudiantes un cuestionario y para los directivos del proyecto por parte de la universidad y de la institución ADINEA, entrevistas, tal como se detalla en la siguiente tabla:

\section{TABLA 2}

Indicadores para medir el impacto de los proyectos de vinculación con la sociedad (Caso carrera de Odontología - UCACUE

\begin{tabular}{|c|c|c|}
\hline Actores & Instrumentos & Indicadores \\
\hline $\begin{array}{l}\text { Pacientes } \\
\text { (representantes) }\end{array}$ & Cuestionario & $\begin{array}{l}\text {-Nivel de participación ciudadana } \\
\text {-Organización } \\
\text {-Utilidad y valor agregado }\end{array}$ \\
\hline Estudiantes & Cuestionario & $\begin{array}{l}\text {-De integración con la comunidad } \\
\text {-Percepción respecto a la planificación y ejecución del } \\
\text { proyecto } \\
\text {-Metas y resultados }\end{array}$ \\
\hline $\begin{array}{c}\text { ADINEA } \\
\text { Directora } \\
\text { Odontólogo }\end{array}$ & Entrevista & $\begin{array}{l}\text {-Nivel de participación ciudadana } \\
\text {-Organización } \\
\text {-Utilidad y valor agregado } \\
\text {-Expectativa }\end{array}$ \\
\hline $\begin{array}{c}\text { Directora } \\
\text { Proyecto } \\
\text { (Universidad) }\end{array}$ & Entrevista & $\begin{array}{l}\text { - Estrategias, técnica y gestión académica } \\
\text {-Trabajo en equipo }\end{array}$ \\
\hline
\end{tabular}

Fuente: Elaboración propia.

Para los beneficiarios directos, que son los pacientes, se planteó un cuestionario a ser llenado por las personas que están bajo su responsabilidad, tomando en consideración la discapacidad de los beneficiarios y las condiciones de prevención sanitaria, ocasionadas por el Coronavirus (Covid 19) al formar parte de los grupos de atención prioritaria, con los indicadores de "Nivel de participación ciudadana" para saber si ellos habían formado parte del proyecto. De igual manera en cuanto al indicador de "Organización", se planteó la calidad de atención que habían recibido, así como si recibieron atención en cuanto a sus preguntas y sugerencias. Finalmente, en el indicador de "Utilidad y valor agregado", se les planteo la inquietud si los servicios recibidos en su salud bucal, mejoraron su calidad de vida, y si este tipo de proyectos son de gran utilidad para su comunidad, además de recabar sugerencias respecto al proyecto.

Los indicadores planteados a los gestores del proyecto, que son los estudiantes participantes del proyecto de vinculación con la sociedad, fueron el de: "Integración con la Comunidad" esto, tanto para constatar si al inicio del proyecto se lo socializo con la comunidad a ser beneficiada, así como para verificar si se receptaron opiniones y sugerencias de las personas a ser beneficiadas, para incluirlas en dicho proyecto. En el indicador de "Percepción respecto a la planificación y ejecución del proyecto" se les plantearon dos interrogantes, una sobre su nivel de satisfacción en cuanto a la planificación y ejecución del proyecto y otra sobre si consideraban que los objetivos planteados en el proyecto se cumplieron o no. De igual manera en el indicador 
de "Metas y resultados" se les planteo, la inquietud, de cuál creerían que sería el nivel de impacto del proyecto en la comunidad a ser implementada, así como si este proyecto mejorará la calidad de vida de dicha comunidad. Finalmente se cogieron las sugerencias de los estudiantes.

En cuanto a las entrevistas planteadas a la directora y odontólogo de la institución beneficiaria, se plantearon los siguientes indicadores: "Nivel de participación ciudadana" y "Organización" para obtener información en cuanto a su participación en la planificación, toma de decisiones y sugerencias en el diseño del proyecto. En el de "Utilidad y valor agregado" para confirmar si el proyecto estuvo de acuerdo a las necesidades de la institución beneficiaria y si el mismo generó un bienestar en los beneficiarios y sus familias, y en el indicador de "Expectativa" se recolectó información sobre la percepción en cuanto a la implementación del proyecto dentro de su institución.

Como último documento de recolección de información, se planteó una entrevista a la directora del proyecto de vinculación, el mismo que consta de dos indicadores; el uno respecto a "Estrategias, técnica y gestión académica" en la cual se plantearon cuatro preguntas, como: los tipos de estrategias utilizadas para vincular a la comunidad al proyecto, también, si se permitió la participación y tomaron en cuenta las sugerencias en el elaboración del diseño del proyecto por parte de la comunidad, y si se realizaron talleres en la institución por parte de la universidad, para socializar el inicio del proyecto. En cuanto al indicador de "Trabajo en equipo" éstas estaban orientadas en evaluar el trabajo en equipo de los estudiantes y de estos con la institución; si el proyecto respondía a las necesidades de la institución beneficiaria y si generó un bienestar en los mismos, así mismo sus expectativas respecto a la implementación del proyecto en la comunidad y algunas sugerencias sobre dicho proyecto.

Para identificar los indicadores que midan el impacto de los procesos de Vinculación con la Sociedad de la carrera de Odontología de la Universidad Católica de Cuenca, desde los indicadores planteados por Del Cioppo \& Bello, se seleccionaron aquellos que nos permitan valorar desde los diversos actores que participaron de manera directa en el proyecto, esto es: los beneficiarios directos del proyecto: pacientes y directivos de la institución beneficiaria y de los gestores del proyecto: estudiantes y directora, para poder tener un percepción amplia del proyecto desde diferentes puntos de vista.

Con los indicadores propuestos se procedió a validarlos, realizando las entrevistas y cuestionarios propuestos. Para los beneficiarios (pacientes) que pertenecen al grupo más alto de vulnerabilidad, que son las personas con discapacidad, y por la difícil accesibilidad a información sobre los mismos, se decidió realizar un muestreo no probabilístico por conveniencia, a las personas encargadas de su cuidado o familiares responsables de los mismos, además de los cuestionarios realizados a la totalidad de la población, de los estudiantes que formaron parte del proyecto y de entrevistas a la directora del proyecto por parte de la universidad, al odontólogo participante por parte de la institución beneficiaria, y finalmente a la presidenta de ADINEA. 
De acuerdo a las encuestas realizadas a los beneficiarios (representantes) en un estudio exploratorio, los principales resultados sobre los indicadores de impacto del proyecto de vinculación analizado, se muestran en la Tabla 3, con sus respectivos porcentajes.

Tabla 3

Beneficiarios (Pacientes)

Porcentaje

Indicador: Nivel de participación ciudadana

Participación en el proyecto de Vinculación

Afirmativo Negativo

$\% 100.00 \quad \% 0.00$

\begin{tabular}{|c|c|c|c|}
\hline Indicador: Organización & Muy Bueno & Bueno & Malo \\
\hline $\begin{array}{l}\text { Calificación a la atención que le brindaron al } \\
\text { representado }\end{array}$ & $\% 58.33$ & $\% 33.33$ & $\% 8.33$ \\
\hline $\begin{array}{l}\text { Fueron escuchadas y atendidas, las preguntas y } \\
\text { sugerencias del representado }\end{array}$ & & $\begin{array}{l}\text { Afirmativas } \\
\% 50.00\end{array}$ & $\begin{array}{l}\text { Negativas } \\
\% 50.00\end{array}$ \\
\hline Indicador: Utilidad y valor agregado & Mucho & Poco & Nada \\
\hline $\begin{array}{l}\text { Servicios recibidos de salud bucal, mejoraron, la } \\
\text { calidad de vida del representado }\end{array}$ & $\% 66.67$ & $\% 25.00$ & $\% 8.33$ \\
\hline $\begin{array}{l}\text { Este tipo de proyectos son de gran utilidad para } \\
\text { comunidad }\end{array}$ & & $\begin{array}{l}\text { Afirmativas } \\
\% 91.67\end{array}$ & $\begin{array}{l}\text { Negativas } \\
\% 8.33\end{array}$ \\
\hline
\end{tabular}

Fuente: Elaboración propia.

Como se puede observar en la tabla 2, en cuanto a la organización se puede analizar que más de la mitad respondieron que fue muy bueno, si sumamos al parámetro de bueno podemos asegurar que los pacientes con discapacidad fueron bien atendidos, y en este mismo indicador en lo referente a si fueron escuchadas las sugerencias de los pacientes, es importante recalcar que la comunicación entre los estudiantes y los pacientes con discapacidad, se vuelve muy complicada, por la situación fisiológica de los segundos, ya que muchos de ellos sufren patologías como el síndrome de down, epóxia neonatal o trastorno heredo genético, entre las principales, de ahí que las opiniones estén divididas en este criterio.

Finalmente en cuanto al indicador de utilidad y valor agregado, un porcentaje bastante elevado consideran que los servicios recibidos de salud bucal mejoraron en mucho, la calidad de vida de los pacientes, esto se reafirma en la siguiente pregunta en donde casi la totalidad de los encuestados afirman que este tipo de proyectos, si son de gran utilidad para la comunidad, afirmación que además es de manera reiterada, y expresadas en las sugerencias, principalmente en la solicitud de que se continúen con este tipo de proyectos en favor de los chicos de ADINEA, o de que deberían ser de manera más continua.

Por otra parte, de los cuestionarios realizados a los 17 estudiantes participantes del proyecto, en la Tabla 4, se pueden observar los porcentajes de los indicadores, que proyecto la investigación.

\section{Tabla 4}


ISSN: 2600-5859

Vol. 4, Nº1.1, p. 124-142, Febrero, 2020

\begin{tabular}{|c|c|c|c|c|}
\hline $\begin{array}{l}\text { Socialización del proyecto con la } \\
\text { comunidad a ser beneficiada }\end{array}$ & & & $\% 94.12$ & $\% 5.88$ \\
\hline $\begin{array}{l}\text { Recepción de opiniones y sugerencias de } \\
\text { las personas beneficiadas, para incluirlas }\end{array}$ & Siempre & A menudo & $\begin{array}{l}\text { Raras } \\
\text { veces }\end{array}$ & Nunca \\
\hline en el diseño del proyecto & $\% 0.00$ & $\% 29.41$ & $\% 29.41$ & $\% 41.18$ \\
\hline $\begin{array}{l}\text { Indicador: Percepción respecto a la } \\
\text { planificación y ejecución del proyecto }\end{array}$ & $\begin{array}{c}\text { Muy } \\
\text { satisfecho }\end{array}$ & Satisfecho & $\begin{array}{l}\text { Poco } \\
\text { satisfecho }\end{array}$ & $\begin{array}{c}\text { Nada } \\
\text { satisfecho }\end{array}$ \\
\hline $\begin{array}{l}\text { Satisfacción con la planificación y } \\
\text { elaboración planteada en el proyecto }\end{array}$ & $\% 52.94$ & $\% 35.29$ & $\% 11.76$ & $\% 0.00$ \\
\hline $\begin{array}{l}\text { Cumplimiento de los objetivos planteados } \\
\text { en el proyecto }\end{array}$ & & & $\begin{array}{c}\text { afirmativas } \\
\% 64.71\end{array}$ & $\begin{array}{r}\text { negativas } \\
\% 35.29\end{array}$ \\
\hline Indicador: Metas y resultados & $\begin{array}{l}\text { Gran } \\
\text { impacto }\end{array}$ & $\begin{array}{l}\text { Buen } \\
\text { impacto }\end{array}$ & $\begin{array}{l}\text { Poco } \\
\text { impacto }\end{array}$ & $\begin{array}{l}\text { Ningún } \\
\text { impacto }\end{array}$ \\
\hline $\begin{array}{l}\text { Nivel de impacto del proyecto en la } \\
\text { comunidad }\end{array}$ & $\% 47.06$ & $\% 47.06$ & $\% 5.88$ & $\% 0.00$ \\
\hline $\begin{array}{l}\text { Proyecto de Vinculación implementado } \\
\text { mejorará la calidad de vida de la }\end{array}$ & $\begin{array}{l}\text { En gran } \\
\text { medida }\end{array}$ & Mucho & Poco & Nada \\
\hline Comunidad & $\% 64.71$ & $\% 29.41$ & $\% 5.88$ & $\% 0.00$ \\
\hline
\end{tabular}

Fuente: Elaboración propia.

En lo referente al indicador de "Integración con la comunidad" la mayoría afirma que, si se realizó la socialización del proyecto con la comunidad, y en lo referente a la recepción de opiniones y sugerencias de los pacientes hacia los estudiantes, existe una división de criterios, aunque el hecho de que "Nunca" mayoritariamente se manifieste, se deba a la falta de comunicación, producto de la discapacidad que sufren los pacientes, algo que ya se manifestó anteriormente. En cuanto al indicador de "Percepción respecto a la planificación y ejecución del proyecto" más de la mitad de los estudiantes manifiestan encontrarse muy satisfechos, y con un porcentaje un poco más alto manifiestan de manera afirmativa, haberse cumplido con los objetivos planteados en el proyecto. En el indicador de "Metas y resultados" los estudiantes manifiestan que el nivel de impacto del proyecto en la comunidad, tuvo un buen y gran impacto y en este mismo indicador sobre si el proyecto mejorará la calidad de vida de la comunidad, se puede observar que lo hará en gran medida.

Para las entrevistas, tanto al Odontólogo como a la Presidenta de ADINEA, se utilizó un solo formato, cuyas principales opiniones, se transcriben en la Tabla 5.

\section{Tabla 5}

Beneficiarios (Directivos)

\section{Indicador: Nivel de participación ciudadana}

¿Se le invitó a usted a participar en la planificación del proyecto por parte de la Universidad?

-Los dos entrevistados manifiestan afirmativamente que sí, y el odontólogo adiciona, que la propuesta del proyecto fue iniciativa suya

¿Participó usted en la toma de decisiones para la elaboración del proyecto?

-La participación en la toma de decisiones estuvo a cargo del odontólogo, por lo que los entrevistados manifiestan que sí

\section{Indicador: Organización}

¿Se tomaron en cuenta sus sugerencias y las de su comunidad para el diseño del proyecto?

-Presidenta manifiesta que sí

-Odontólogo que, de manera parcial, ya que faltaron muchas cosas por hacer 
ISSN: 2600-5859

Indicador: Utilidad y valor agregado

¿Considera usted que el proyecto está de acuerdo a las necesidades de su comunidad?

-Presidenta manifiesta que sí

-Odontólogo que no, ya que falto más practica por parte de los estudiantes

¿Cree usted que este proyecto generará un bienestar en los beneficiarios y sus familiares?

-De manera unánime manifiestan que sí, ya que eso es lo que se pretendió y fue muy bien visto por los padres de familia de los pacientes.

Indicador: Expectativa

¿Qué expectativas tiene usted respecto a la implementación de este proyecto en ADINEA?

-Presidenta, que siempre están buscando este tipo de proyectos, porque son muy positivos

-Odontólogo, que los estudiantes de último año realicen sus prácticas en la institución.

Sugerencias:

-Presidenta, manifiesta que el proyecto se terminó y no se lo continuó, por lo que se lo debería reactivar

-Odontólogo, los estudiantes tienen la posibilidad de hacer sus prácticas con pacientes con discapacidad

Fuente: Elaboración propia.

Si bien es cierto en varias preguntas a los dos entrevistados existen concordancia, sin embargo, en aquellas que existen diferencias, éstas son propias de dos perspectivas diferentes, en las cuales se desempeñan cada uno de ellos, el uno desde su posición de odontólogo que es más cercana a la parte práctica del proyecto, y la otra desde la parte administrativa.

A la directora del proyecto de vinculación con la sociedad, también se le realizó una entrevista, pero tomando en consideración los indicadores planteados en necesidades de información de la Tabla 1, y cuyos resultados transcribimos en la Tabla 6.

\section{Tabla 6}

Gestores (Directora del proyecto)

\section{Indicador: Estrategias, técnica y gestión académica}

¿Qué tipos de estrategias se utilizaron para vincular a la comunidad al proyecto?

- Se trabajó con el odontólogo de turno del colegio. Fue una labor en conjunto

¿Se permitió la participación de la comunidad en la toma de decisiones para la elaboración del proyecto?

- Se pidió directivas a la dirección del colegio

¿Se tomaron en cuenta las sugerencias de la comunidad para el diseño del proyecto? ¿Cuáles?

- Sí, el tiempo de atención; los alumnos debieron ser de últimos ciclos para poder atender a los pacientes

¿Se realizaron talleres en la institución, por parte de la Universidad, para socializar el inicio del proyecto?

- Sí, el primer taller fue la presentación del equipo y de los objetivos que queríamos alcanzar

\section{Indicador: Trabajo en equipo}

¿Cómo evalúa el trabajo en equipo por parte de los estudiantes y de estos con la comunidad?

-Bueno

¿Considera usted que el proyecto está de acuerdo a las necesidades de la comunidad? -Sí

¿Considera que la realización de este proyecto generó un bienestar en los beneficiarios?

- Sí, los niños atendidos se sintieron cómodos con nosotros y los alumnos de pregrado

aprendieron de los pacientes 
¿Qué expectativas tiene usted respecto a la implementación de este proyecto en la comunidad?

- Es necesario educar a los alumnos de pregrado para el manejo y atención del paciente con discapacidad

Sugerencias: Ninguna

Fuente: Elaboración propia.

De los indicadores planteados a la directora del proyecto de vinculación con la sociedad, se pueden desprender algunos criterios positivos utilizados en cuanto a estrategias de socialización y participación, sobre todo en el trabajo en equipo y en conjunto con ADINEA.

\section{Conclusiones}

- La gran mayoría de universidades ecuatorianas no realizan evaluaciones posteriores a la realización de sus proyectos de $\mathrm{VcS}$, ya que el marco jurídico ecuatoriano no lo exige, sin embargo, esto impide avanzar en el mejoramiento de los proyectos universitarios de $\mathrm{VcS}$, y plantearnos interrogantes respecto como están funcionando, sus niveles de organización, estrategias de planificación y participación, trabajo en equipo, metas y la utilidad y valor agregado que estos proyectos están brindando a las comunidades o instituciones en donde se los implementa, de estas interrogantes surgen precisamente los indicadores planteados en la presente investigación y que nos permitieron evaluar en dichos parámetros el proyecto de $\mathrm{VcS}$, en el caso de la carrera de Odontología de la Universidad Católica de Cuenca..

- Del análisis teórico y bibliográfico realizado, sobre la evaluación de los proyectos de vinculación implementados por las universidades ecuatorianas, se visualiza la importancia de la evaluación del impacto generado por el proyecto en la comunidad o institución implementada, dicha evaluación de impactos debe ir más allá de una evaluación de cumplimiento de metas y objetivos. Además, las estadísticas que proyectan las evaluaciones a nivel de indicadores, nos permiten obtener una idea real, del aporte de las universidades a las problemáticas de las comunidades e instituciones beneficiarias, y retroalimentar los proyectos a implementarse a futuro.

- De los resultados obtenidos con los indicadores propuestos, en la evaluación del impacto del proyecto de $\mathrm{VcS}$, denominado: "Mejoramiento de la salud bucal del paciente con discapacidad", de la carrera de odontología, se puede observar una buena planificación y elaboración del proyecto desde la universidad con la institución beneficiaria. Así mismo el impacto positivo que genero el proyecto, en el mejoramiento de la calidad de vida de los beneficiarios directos y de igual manera la concientización de la importancia de apoyar a los grupos más vulnerables de nuestra sociedad, por parte de los estudiantes participantes, a más de haber realizado sus prácticas preprofesionales.

- Los indicadores propuestos para medir el impacto del proyecto: "Mejoramiento de la salud bucal del paciente con discapacidad", están planteados para que puedan medir otros proyectos de $\mathrm{VcS}$ de la carrera de medicina y de aquellos proyectos, donde se puedan medir las variables de los beneficiarios directos, y sobre todo el nivel de articulación y planificación del proyecto de la universidad, con la comunidad o instituciones beneficiarias. 


\section{Referencias bibliográficas.}

Álava, L., Moreira, T y Viteri, R. (2019). Experiencias de vinculación con la sociedad en la comunidad Santa Martha, parroquia Río Chico del Cantón Portoviejo, provincia de Manabí. Revista Caribeña de Ciencias Sociales (junio 2019). Recuperado de https://www.eumed.net/rev/caribe/2019/06/vinculacion-sociedad-

comunidad.html//hdl.handle.net/20.500.11763/caribe1906vinculacion-sociedadcomunidad

Barreno, M., Barreno, Z., y Olmedo, A. (2018). La educación superior y su vinculación con la sociedad: referentes esenciales para un cambio. Universidad y Sociedad, 10(3), 40-45. Recuperado de http:// rus.ucf.edu.cu/index.php/rus

Brito, L., Quezada, C., y Gordillo, I. (2018 a). La planificación de la vinculación con la sociedad, una condición impostergable de legitimación. Caso: Universidad Técnica de Machala, Ecuador. Redes 2017, 71-90. Recuperado de http://repositorio.utmachala.edu.ec/handle/48000/14292

Brito, L., Quezada, C., y Gordillo, I. (2018 b). La vinculación con la sociedad y la universidad pública en el Ecuador. Redes 2017, 28-53. Recuperado de http://repositorio.utmachala.edu.ec/bitstream/48000/12492/1/LaUniversidad-yLaVinculacionConLaSociedad.pdf

Brito, L., Gordillo, I., y Quezada, C. (2016). La vinculación con la sociedad y la universidad pública en el Ecuador. Revista Contribuciones a las Ciencias Sociales. Recuperado de http://www.eumed.net/rev/cccss/2016/03/vinculacion.html

Castro, M., Borroto, O., y Almuiñas, J. (2018). Extensión universitaria y desarrollo local: Una perspectiva en construcción. 24(1), 16-23. Recuperado de http://revista.sangregorio.edu.ec/index.php/Revistasangregorio/article/view/737

Cedeño, R. Ostaíza, J. Vélez, G y Solórzano, K. (2018) Vinculación con la sociedad: Un desafío posible desde el altruismo, el compromiso y la organización. Revista San Gregorio, 24(1), 60-67. Recuperado de http://revista.sangregorio.edu.ec/index.php/Revistasangregorio/article/view/415

Chávez, G. (2016). Universidad, Vinculación Con La Sociedad Y Procesos De Mejoramiento. Quipukamayoc Revista de la Facultad de Ciencias Contables. 24(45), 15-22. Recuperado de https://www.researchgate.net/publication/319660994_Universidad_Vinculacion_Con_ La_Sociedad_y_Procesos_de_Mejoramiento

Creswell, J. W. (2009). Research Design: qualitative, quantitative, and mixed methods approaches (3.a ed.). Londres: Sage.

De Aparicio, X., Chininin, M., y Toledo, O. (2017). El rol de la vinculación en la integración de las funciones sustantivas de la Universidad Metropolitana del Ecuador. Universidad y Sociedad, 9(4), 37-43. Recuperado de http://rus.ucf.edu.cu/index.php/rus 
Del Cioppo, J y Bello, M. (2018). Indicadores de impacto social para evaluación de proyectos de vinculación con la colectividad. Económicas CUC, 39(1). 105-116. DOI: http://dx.doi.org/10.17981/econcuc.39.1.2018.07

Hermida, C. (2017). Vinculación con la sociedad y universidad saludable. Rev Fac Cien Med (Quito), 2017; $42 \quad$ (1), 150-155. Recuperado de http://revistadigital.uce.edu.ec/index.php/Ciencias_Medicas/article/view/1576

Herrera, K. (2016). Vínculo Entre Universidad y Sociedad: Una Mirada Desde los Estudios Sociales de Ciencia y Tecnología en el Ecuador. Memorias del segundo Congreso Internacional de Ciencias Pedagógicas: Por una educación innovadora, para un desarrollo humano sostenible. 1849-1858. Recuperado de https://dialnet.unirioja.es/servlet/articulo?codigo=7199792

Ley Orgánica de Educación Superior (LOES), (12 de octubre de 2010). Recuperado de Recuperado de http://www. ces. gob. ec/descargas/ ley-organica-de-educacion-superior

Malagón, L. (2006). La Vinculación Universidad-Sociedad desde una perspectiva social. Educación y Educadores, 9(2), 79-93. Recuperado de https://educacionyeducadores.unisabana.edu.co/index.php/eye/article/view/664/749

Maldonado, B., Buenaño, J., y Benavides, K. (2018). Aproximación a un Modelo de Gobernanza del Sistema de Educación Superior Ecuatoriano. Revista San Gregorio, 24(1), 78-88. Recuperado de http://revista.sangregorio.edu.ec/index.php/REVISTASANGREGORIO/article/view/7 07

Mendoza, C., Jácome, T y Vélez, M. (2017). La Participación de la Comunidad Universitaria y su Vinculación con la Sociedad. Revista Científica Hallazgos 21, 2(3), 212 221.Recuperado de https://revistas.pucese.edu.ec/hallazgos21/article/view/192

Pérez, A. (2018). Relación universidad y sociedad en América Latina: un cauce a través de la responsabilidad social universitaria. Redes 2017, 54-70. Recuperado de http://repositorio.utmachala.edu.ec/handle/48000/14291

Pérez, I. (2018). Educación para el Desarrollo Sostenible más allá del currículo escolar. Reflexiones socio-culturales. Revista San Gregorio, 24(1), 140-148. Recuperado de http://revista.sangregorio.edu.ec/index.php/Revistasangregorio/article/view/415

Quezada, C., Carmenate, L., y Brito, L. (2018). La globalización y su efecto en las universidades estatales del Ecuador. Redes 2017, 14-21. Recuperado de http://repositorio.utmachala.edu.ec/bitstream/48000/14289/1/Cap.1-

La\%20globalizaci\%C3\%B3n\%20y\%20su\%20efecto\%20en\%20las\%20universidades $\% 20$ estatales\%20del.pdf

Riofrío, O., Serrano, B., y Sotomayor, G. (2018). La Vinculación con la sociedad, un desafío de transformación de la Educación Superior en la sociedad de la información y del 
ISSN: 2600-5859

conocimiento.

Redes

2017

91-105.

Recuperado

de

http://repositorio.utmachala.edu.ec/handle/48000/14293

Rueda, I., Acosta, B., y Cueva, F. (2020). Las Universidades y sus Prácticas de Vinculación con la Sociedad. Educ. Soc. vol.41. Recuperado de https://www.scielo.br/scielo.php?script=sci_arttext\&pid=S0101-73302020000100305

Serrano, C., y Condor, V. (2018). La educación a distancia y la vinculación con la sociedad. Análisis de modalidad a distancia de la Universidad Nacional de Loja. Universidad y Sociedad, 10(3), 241-246. Recuperado de http://rus.ucf.edu.cu/index.php/rus

Simbaña. H., y Correa, L. (2017). La vinculación con la sociedad en el Ecuador: Reflexiones sobre su itinerario curricular. Vínculos-ESPE, 2(1), 5-12. Recuperado de https://journal.espe.edu.ec/ojs/index.php/vinculos/article/view/563/777

Torres, M., Escalante, A., y García, N. (2020). Propuesta de indicadores para medir vinculación universitaria: Mirada de funcionarios de instituciones de educación superior públicas. Archivos Analíticos de Políticas Educativas, 28(65). https://doi.org/10.14507/epaa.28.4333

Viña, N., Sacoto, V. y Landívar, J. (2019). Contribución de la Universidad ecuatoriana en beneficio de sectores vulnerables - Vinculación con la Sociedad. Espacios, 40(23), 11. Recuperado de http://www.revistaespacios.com/a19v40n23/a19v40n23p11.pdf

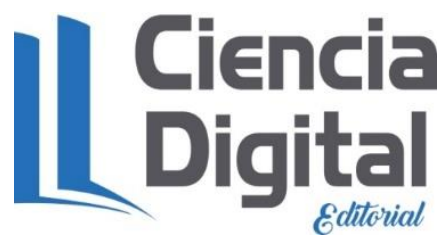




\section{PARA CITAR EL ARTÍCULO INDEXADO.}

Quezada Ortega, J. R., Banegas Campoverde, C. M., \& Castillo Ortega, Y. (2021). Evaluación del impacto de la vinculación con la sociedad en el caso de la carrera de Odontología de la Universidad Católica de Cuenca. ConcienciaDigital, 4(1.1), 124-142. https://doi.org/10.33262/concienciadigital.v4i1.1.1550

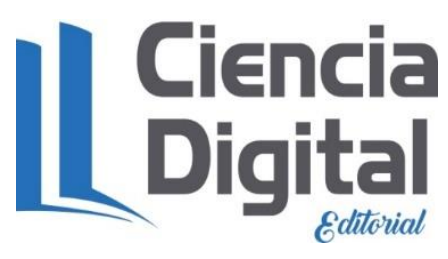

El artículo que se publica es de exclusiva responsabilidad de los autores y no necesariamente reflejan el pensamiento de la Revista Conciencia Digital.

El artículo queda en propiedad de la revista y, por tanto, su publicación parcial y/o total en otro medio tiene que ser autorizado por el director de la Revista Conciencia Digital.
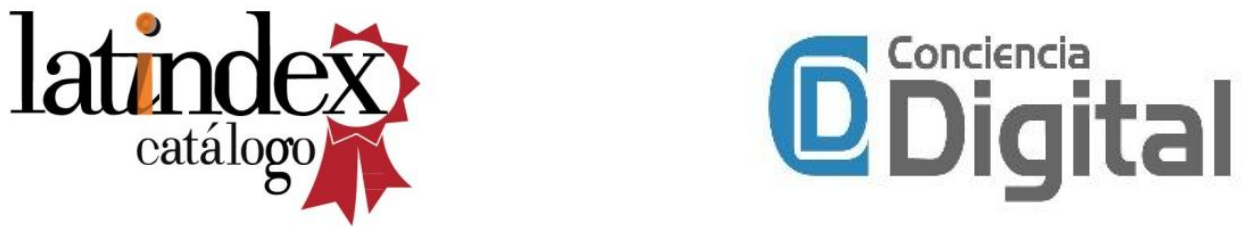